\title{
Epileptic Encephalopathies: An Overview
}

\author{
Sonia Khan ${ }^{1}$ and Raidah Al Baradie ${ }^{2}$ \\ ${ }^{1}$ Department of Clinical Neurosciences, Prince Sultan Military Medical City, P.O. Box 7897, Riyadh 11159, Saudi Arabia \\ ${ }^{2}$ Department of Pediatrics, University of Dammam and King Fahad Specialist Hospital, P.O. Box 2435, Dammam 31451, Saudi Arabia
}

Correspondence should be addressed to Sonia Khan, ecirera@rmh.med.sa

Received 25 February 2012; Accepted 12 September 2012

Academic Editor: Nicola Specchio

Copyright (C) 2012 S. Khan and R. Al Baradie. This is an open access article distributed under the Creative Commons Attribution License, which permits unrestricted use, distribution, and reproduction in any medium, provided the original work is properly cited.

Epileptic encephalopathies are an epileptic condition characterized by epileptiform abnormalities associated with progressive cerebral dysfunction. In the classification of the International League Against Epilepsy eight age-related epileptic encephalopathy syndromes are recognized. These syndromes include early myoclonic encephalopathy and Ohtahara syndrome in the neonatal period, West syndrome and Dravet syndrome in infancy, myoclonic status in nonprogressive encephalopathies, and LennoxGastaut syndrome, Landau-Kleffner syndrome, and epilepsy with continuous spike waves during slow wave sleep in childhood and adolescences. Other epileptic syndromes such as migrating partial seizures in infancy and severe epilepsy with multiple independent spike foci may be reasonably added. In this paper, we provide an overview of epileptic encephalopathies including clinical neurophysiological features, cognitive deterioration, and management options especially that these conditions are generally refractory to standard antiepileptic drugs.

\section{Introduction}

Epileptic encephalopathy is defined as a condition in which epileptiform abnormalities are believed to contribute to the progressive disturbance in cerebral function, but this definition may be ambiguous [1]. The report of the International League Against Epilepsy (ILAE) Task Force on classification and terminology includes 8 syndromes under epileptic encephalopathies: early myoclonic encephalopathy, Ohtahara syndrome, West syndrome, Dravet syndrome, myoclonic status in nonprogressive encephalopathies, Lennox-Gastaut syndrome, Landau-Kleffner syndrome, and epilepsy with continuous spike waves during slow-wave sleep [1]. To these syndromes, the migrating partial seizures in infancy and severe epilepsy with multiple independent spike foci may be reasonably added [2].

A common feature is that these disorders are usually refractory to standard antiepileptic drugs (AEDs) [3]. As a result, more aggressive use of AEDs considered effective in suppressing interictal epileptiform discharges (e.g., benzodiazepines, valproic acid, and lamotrigine), immunomodulatory therapies (e.g., corticosteroids, intravenous immunoglobulin (IVIG), and plasmapheresis), ketogenic diet, and surgical options is often considered [3]. In this paper, epileptic encephalopathies will be dealt with in the following concept: a particular group of usually agerelated and extremely intractable epilepsies with characteristic generalized minor seizures and massive epileptic EEG abnormalities, both of which cause stagnation or deterioration in mental and cognitive functions in addition to the preexisting developmental deficit due to organic brain damage [1-3].

\section{Epileptic Encephalopathy Syndromes in Infancy}

2.1. Early Infantile Epileptic Encephalopathy (Ohtahara Syndrome). Ohtahara syndrome is the earliest form of the agedependent neonatal epileptic encephalopathies and was first described by Ohtahara and colleagues in 1976 [4]. It is often defined as "Early Infantile Epileptic Encephalopathy (EIEE) with burst-suppression" or "early myoclonic encephalopathy (EME)" [4]. Symptoms appear within the first 3 months of birth and usually within the first 10 days. Often symptoms will appear with the first few hours after birth, and in some cases mothers have felt possible seizures activity in utero. Onset is acute in previously normal children [4]. 
Main seizure pattern is tonic spasms; other patterns include tonic/clonic, clonic, myoclonic, atonic, absences, partial, complex partial (with or without secondary generalization), gelastics, and Jacksonians. Seizures can appear in clusters or singly and patterns are likely to change with time. It is not uncommon for patterns to reappear at a later stage [4]. EEG pattern is characterized as burst suppression during both waking and sleeping states. This means that the EEG (electroencephalogram) tends to show periods of very little electrical brain activity followed by a burst of high spiky activity before returning to very low activity again. Sometimes, one side of the brain seems to be affected more than the other [5]. Seizures are intractable; although in some cases they can be improved through treatment. In general prognosis is poor with severe psychomotor retardation and significant learning difficulties. Frequently cases will progress to West syndrome or partial epilepsy (usually during infancy). Later a much smaller number develops to Lennox-Gastaut syndrome. Psychomotor development may be slightly better if the infants do not develop West or Lennox-Gastaut syndrome. Half of the children are likely to die in infancy or childhood $[5,6]$. Although the disorder in incurable, much can be done to improve the lives not only of the children but also of the families. Seizure control is the main aim and will be attempted either through optimized dosages of anticonvulsants such as vigabatrin (Topamax), Dilantin, Zonegran, and Phenobarbitone, or through steroid therapies using ACTH and Prednisone. AEDs can be taken in either mono- or polytherapies. The quest for seizure control can be a slow and frustrating process. There is also the possibility of utilizing such treatments as the ketogenic diet, the VNS, or more invasive surgery, such as a partial resection or complete hemispherectomy. Physiotherapy and occupational therapies can help improve motor skills, while hippotherapy can help improve general mobility, strength, and endurance [7].

2.2. Early Myoclonic Encephalopathy. Early myoclonic encephalopathy, an epileptic syndrome with onset either in the neonatal period or the first months of life, is characterized by erratic, fragmentary, or massive myoclonus, partial seizures, and late tonic spasms. The prognosis is severe. Early myoclonic encephalopathy is characterized clinically by the onset of erratic or fragmentary myoclonus. Other types of seizures, including simple partial seizures, massive myoclonia, and tonic spasms, can also occur. Erratic, partial myoclonus usually appears as the first seizure, even as early as a few hours after birth. The myoclonus usually involves the face or extremities and may be restricted to an eyebrow, a single limb, or a finger. The jerks occur when infants are awake or asleep, and they are often described as "erratic" because they shift typically from one part of the body to another in a random, asynchronous fashion.

Frequency varies from occasional to almost continuous. In addition to limited partial myoclonus, generalized myoclonus may also be observed occasionally in some cases. Partial seizures are frequent and occur shortly after erratic myoclonus. The semiology of partial seizures is subtle, consisting, for instance, of eye deviation or autonomic phenomena such as apnea or flushing of the face. Tonic seizures are reported frequently and can occur in the first month of life or afterwards; they may occur both in sleep and wakefulness. From a clinical standpoint, the child presents a diffuse tonic contraction, usually extending to the extremities. Real epileptic spasms are rare and generally appear later. Neurologic abnormalities are constant: very severe delay in psychomotor acquisitions, marked hypotonia, and disturbed alertness, sometimes with vegetative state. Signs of peripheral neuropathy may also occur in rare cases [8]. Early myoclonic encephalopathy is believed to have various prenatal etiologies that often remain unknown. Some conditions, such as inborn error of metabolism, can produce the clinical and EEG picture typical of early myoclonic encephalopathy such as nonketotic hyperglycinemia, D glyceric acidemia, propionic acidemia, molybdenum cofactor deficiency, and methylmalonic acidemia. Few patients present with a clinical picture similar to early myoclonic encephalopathy with atypical burst-suppression pattern that recover completely after pyridoxine therapy. Some malformative disorders can also cause early myoclonic encephalopathy, but more often they produce Ohtahara syndrome [9]. In early myoclonic encephalopathy, EEG is characterized by a "burst-suppression" pattern with bursts of spikes, sharp waves, and slow waves, which are irregularly intermingled and separated by periods of electrical silence (Figure 1). The EEG paroxysms may be either synchronous or asynchronous over both hemispheres. There is no normal background activity. The burst-suppression pattern usually evolves into atypical hypsarrhythmia or into multifocal paroxysms after 3 to 5 months of life. Erratic myoclonus does not generally have an ictal EEG counterpart. Partial seizures have EEG characteristics similar to those of neonatal fits. The CT and MR findings vary and are related to etiology. The brain may be either grossly normal or have asymmetrical enlargement of one hemisphere, dilatation of the corresponding lateral ventricle, or cortical and periventricular atrophy [10]. Considering the inborn error of metabolism reported above, the serum levels of amino acids should be determined, especially glycine and glycerol metabolites and organic acids, as well as the amino acids in the cerebrospinal fluid $[4,8,9]$. The prognosis for early myoclonic encephalopathy is poor and there is no effective therapy for early myoclonic encephalopathy $[9,10]$.

2.3. Infantile Spasms (West Syndrome). West syndrome usually occurs in the first year of life and consists of the triad of infantile spasms, developmental deterioration, and a hypsarrhythmia pattern on EEG [11]. The epileptic spasms are brief, generalized seizures involving extension and/or flexion axially, and of the extremities. An individual spasm lasts for seconds, often longer than typical myoclonic seizures, though not as long as most tonic seizures. The spasms may be subtle and may be isolated at onset, typically clustering later in the course. Several clusters per day, particularly in drowsiness, are characteristic $[11,12]$. Hypsarrhythmia, the typical interictal EEG finding, consists of a disorganized 


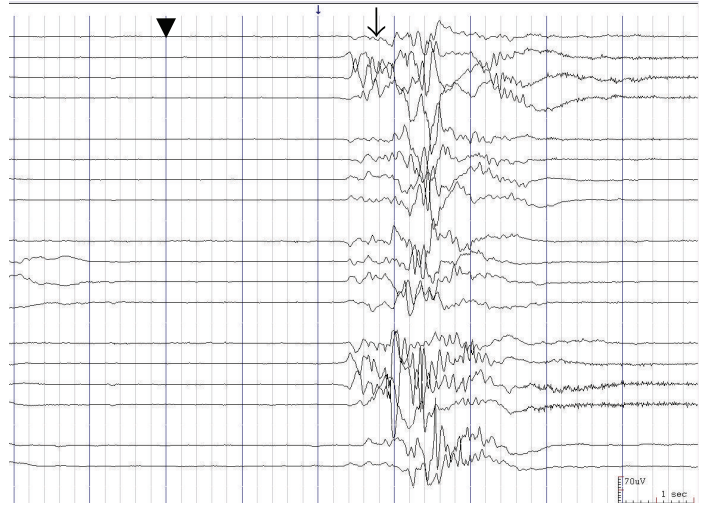

FIGURE 1: EEG is characterized by a "burst-suppression" pattern with bursts of spikes, sharp waves, and slow waves (arrow), which are irregularly intermingled and separated by periods of electrical silence (arrow head).

pattern with asynchronous, very high amplitude slowing and frequent multifocal spike and sharp wave discharges (Figure 2). The ictal EEG typically reveals a generalized slow wave followed by a diffuse voltage attenuation (electrodecrement) (Figure 3), which may associate with a spasm or be only electrographic (without clinical correlation) [12]. No clear etiology is found in approximately $40 \%$ of cases. There is a broad range of potential causes, including cerebral malformations, infection, hemorrhage, hypoxicischemic injury, metabolic disorders, and genetic conditions, such as Down syndrome [12, 13]. Variation in studying methodologies prohibits a clear recommendation for first line treatment; however, ACTH and vigabatrin are usually used in practice. Corticosteroids may be less efficacious than ACTH, although they are effective. Vigabatrin may be more efficacious in tuberous sclerosis. Other agents that are efficacious include valproate, levetiracetam, topiramate, zonisamide, lamotrigine, and benzodiazepines [11]. The ketogenic diet is helpful in most cases [12]. Focal cortical resection or hemispherectomy may be considered for cases that are lesional and medically intractable [11-13]. Development remains unaffected only in a minority. Most children experience slowing, plateauing, or regression of their developmental trajectory. The developmental prognosis partially depends on the etiology. No specific AED has been shown to affect long-term developmental outcome. An extensive literature review revealed that $16 \%$ had normal development, and $47 \%$ had continued seizures at an average followup of 31 months. When classified by etiology, normal development was described in $51 \%$ of cryptogenic cases versus only $6 \%$ of symptomatic cases. Approximately $17 \%$ of cases evolved into Lennox-Gastaut syndrome [12].

2.4. Malignant Epilepsy with Migrating Partial Seizures in Infancy. Onset of this rare syndrome occurs in the first year of life and may occur in the neonatal period. It is characterized by frequent partial seizures of multifocal onset, with autonomic or motor involvement. The seizures increase in frequency and may become near continuous. Clinical

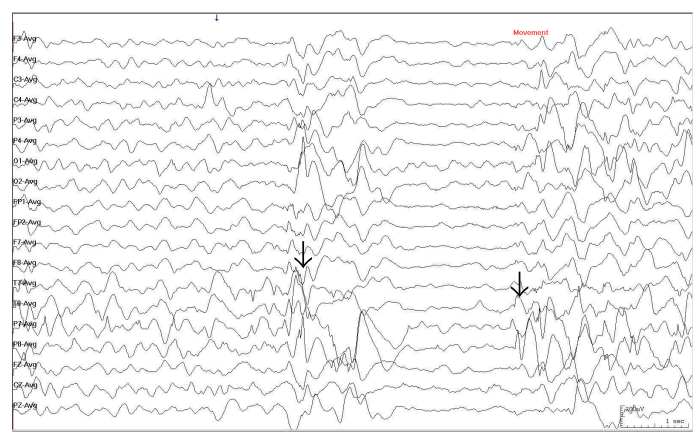

FIGURe 2: Hypsarrhythmia, the typical interictal EEG finding, consists of a disorganized pattern with asynchronous, very high amplitude slowing and frequent multifocal spike and sharp wave discharges (arrows).

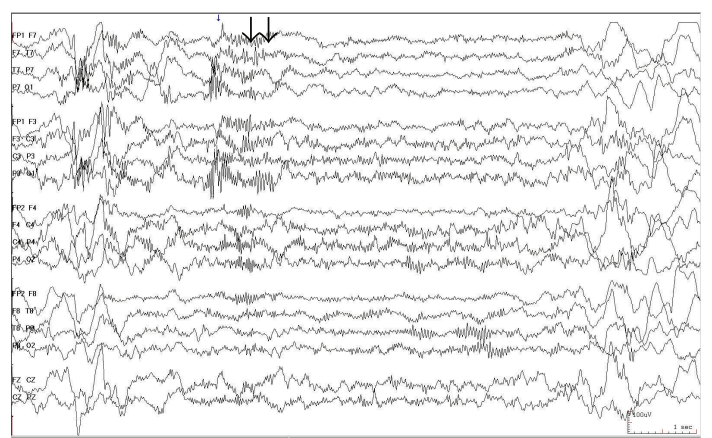

FIgURE 3: The ictal EEG in West syndrome typically reveals a generalized slow wave followed by diffuse voltage attenuation (electrodecrement), which may associate with a spasm or be only electrographic without clinical correlate (arrows).

semiology of the seizures include lateral head and eyes deviation, focal clonic seizures of the eyes, face or limbs, unilateral or bilateral focal tonic seizures, automatic movements such as chewing, mastication, autonomic features such as apnea, salivation or facial flushing and secondary generalized tonic clonic seizures. The interictal EEG reveals multifocal epileptiform activity and slowing, diffuse slowing of the background activity. A few patients may have a normal EEG. Then the EEG background activity became slow with fluctuating asymmetry between different recordings. Initially sleep-waking cycle can be identified; spindles are rare and asymmetric. The ictal EEG confirms multifocal onsets, which may shift from seizure to seizure. In most cases, there is no clear etiology or structural problems, suggesting that genetic factors may be causative or contributory. Seizures are often difficult to control with standard AEDs. Bromides, stiripentol, and clonazepam may be helpful in some cases. Developmental regression is common, and death has been reported in infancy and childhood in severe cases [14].

\subsection{Myoclonic Status in Nonprogressive Encephalopathies.} This rarely reported disorder has onset in infancy or early childhood, with onset usually during the first year of life. Seizures typically begin with partial motor seizures, although 
myoclonic status may occur at onset. Myoclonic absences, massive myoclonias, and rarely generalized or hemiclonic seizures may occur. Myoclonias may be multifocal and occur with startles. Myoclonic status epilepticus may be recurrent. Motor abnormalities and movement disorders are common. The interictal EEG consists of multifocal epileptiform discharges and background slowing. Epileptiform discharges are potentiated in sleep, in some cases similar to an ESES pattern. Ictal EEG recording may demonstrate generalized slow spike and wave, or an absence pattern, depending on the seizure type. A genetic cause is identifiable in approximately half of children, including Angelman syndrome and $4 \mathrm{p}$ syndrome. Other reported causes include hypoxic-ischemic injury and cortical dysplasia. Episodes of myoclonic status may respond to benzodiazepines. AEDs that may be efficacious include valproate with ethosuximide or clobazam. Children have a poor prognosis, experiencing developmental regression and eventual severe mental retardation. The repeated episodes of myoclonic status may contribute to cognitive deterioration [15].

2.6. Severe Myoclonic Epilepsy in Infancy (SMEI) Dravet Syndrome. This is an uncommon form of childhood epilepsy. Out of 500 children with epilepsy, only one, or at most two, child is likely to have this form of epilepsy. It is also called Dravet syndrome. The epilepsy starts with seizures which may not initially differ from those associated with feverish illnesses. This syndrome tends to develop during the second year of life. It may not be possible to make this diagnosis until the child is two, three, or even four years old. The seizures begin in the first year of life. They are most often associated with high temperatures and often just involve one side of the body, although both sides of the body may be involved. The seizures are characterized by jerking rather than stiffness and jerking. They often recur quite frequently in the first year of life. However, at this time it is not easy to differentiate these children from others with febrile convulsions who get better and who do not go on to develop other types of seizure. During the second year of the life of children with SMEI, seizures become more frequent and persistent, are often more obviously partial (involving one part of the body), and no longer occur when a child has a high temperature but at any time of day and night. In addition to the partial seizures are the myoclonic jerks. Often the children are photosensitive (have seizures brought on by flashing lights). Seizures may also sometimes be brought on by hot environments or hot showers or baths. The early development of affected children is usually normal but once the myoclonic seizures and partial seizures start in their second year of life, children may lose skills or their developmental progress may slow down. The child's speech and language may be particularly affected. The electroencephalogram (EEG) which records the electrical activity in the brain is usually normal early in this condition. Later, by the time the child is 18 months old, there is evidence of epileptic activity with spike and wave or polyspike discharges, which occur either as single event or in bursts. These may be generalized involving the whole brain or occurring just from on small area of the brain.
Some children show EEG evidence of sensitivity to flashing lights but this does not occur in all. Brain scans are usually normal. More recently a specific genetic abnormality, called a "mutation", has been found in at least 70 per cent of children with SMEI. This mutation is known as the "SCN1A" mutation. It is likely that other mutations will also be found in children with SMEI. The mutation can be looked for in a simple blood test and this has been very helpful in making or confirming a diagnosis of this epilepsy syndrome. SMEI is very resistant to anti-epileptic drugs. Phenobarbital, sodium valproate (Epilim), and lamotrigine (Lamictal) are usually tried first. However, lamotrigine may actually make the myoclonic seizures worse in many children. Other options include a medication called stiripentol, topiramate (Topamax), clonazepam (Rivotril), and clobazam (Frisium). A combination of sodium valproate with either topiramate or stiripentol may be the most helpful. A short course of a steroid (called prednisolone) and the ketogenic diet may also be helpful. Because children with SMEI always have learning difficulties, they will also need full educational assessment and support $[16,17]$.

\section{Epileptic Encephalopathy Syndromes in Childhood}

3.1. Lennox-Gastaut Syndrome LGS. Childhood epileptic encephalopathy, or Lennox-Gastaut syndrome (LGS), is a devastating pediatric epilepsy syndrome constituting 1-4\% of childhood epilepsies. The syndrome is characterized by multiple seizure types; mental retardation or regression; abnormal findings on electroencephalogram (EEG), with paroxysms of fast activity and generalized slow spike and wave discharges $(1.5-2 \mathrm{~Hz})$ (Figure 4$)$. The most common seizure types are tonic-axial, atonic, and absence seizures, but myoclonic, generalized tonic-clonic, and partial seizures can be observed (see clinical presentation). An EEG is an essential part of the workup for LGS. Neuroimaging is an important part of the search for an underlying etiology. LGS can be classified according to its suspected etiology as either idiopathic or symptomatic. Patients may be considered to have idiopathic LGS if normal psychomotor development occurred prior to the onset of symptoms, no underlying disorders or definite presumptive causes are present, and no neurologic or neuroradiologic abnormalities are found. In contrast, symptomatic LGS is diagnosed if a likely cause can be identified as being responsible for the syndrome. Population-based studies have found that $70-78 \%$ of patients with LGS have symptomatic LGS. Underlying pathologies in these cases include encephalitis and/or meningitis, tuberous sclerosis, brain malformations (e.g., cortical dysplasias), birth injury, hypoxia-ischemia injury, frontal lobe lesions and trauma. Overall, LGS accounts for $1-4 \%$ of patients with childhood epilepsy but $10 \%$ of patients with onset of epilepsy when younger than 5 years. The prevalence of LGS in Atlanta, GA, USA, was reported as 0.26 per 1000 live births. LGS is more common in boys than in girls. The prevalence is 0.1 per 1000 population for boys, versus 0.02 per 1000 population for girls (relative risk, 5.31). The 


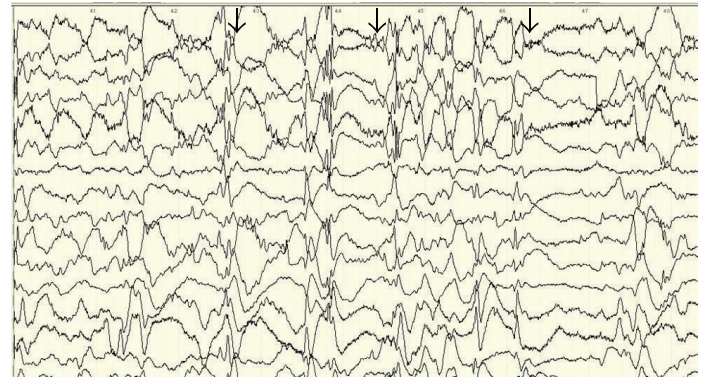

FIGURE 4: EEG in Lennox-Gastaut syndrome with paroxysms of fast activity and generalized slow spike and wave discharges $(1.5-2 \mathrm{~Hz})$ (arrows).

mean age at epilepsy onset is $26-28$ months (range, $1 \mathrm{~d}$ to $14 \mathrm{y}$ ). The peak age at epilepsy onset is older in patients with LGS of an identifiable etiology than in those whose LGS has no identifiable etiology. The difference in age of onset between the group of patients with LGS and a history of West syndrome (infantile spasm) and those with LGS without West syndrome is not significant. The average age at diagnosis of LGS in Japan was 6 years (range, 215 y). Epidemiologic studies in industrialized countries (e.g., Spain, Estonia, Italy, and Finland) have demonstrated that the proportion of epileptic patients with LGS seems relatively consistent across the populations studied and similar to that in the United States. The prevalence of LGS is $0.1-0.28$ per 1000 population in Europe. The annual incidence of LGS in childhood is approximately 2 per 100,000 children. Among children with intellectual disability, $7 \%$ have LGS, while $16.3 \%$ of institutionalized patients with intellectual disability have LGS. Long-term prognosis overall is unfavorable but variable in LGS. Longitudinal studies have found that a minority of patients with LGS eventually could work normally, but $47-76 \%$ still had typical characteristics (mental retardation, treatment-resistant seizures) many years after onset and required significant help (e.g., home care, institutionalization). A variety of therapeutic approaches are used in LGS, ranging from conventional antiepileptic agents to diet and surgery. Unfortunately, much of the evidence supporting these approaches is not robust, and treatment is often ineffective. The medical treatment options for patients with LGS can be divided into the following 3 major groups: The medical treatment options for patients with LGS include the use of antiepileptic drugs such as valproic acid and benzodiazepines such as clonazepam, nitrazepam and clobazam, vigabatrin, zonisamide, lamotrigine, topiramate and rufinamide proven effective by double-blind placebocontrolled studies (e.g., lamotrigine, topiramate, felbamate, and rufinamide). The ketogenic diet may be useful in patients with LGS refractory to medical treatment. Surgical options for LGS include corpus callosotomy, vagus nerve stimulation, and focal cortical resection [18-21].

3.2. Electrical Status Epilepticus during Slow Sleep (ESES). A disorder in which sleep induced an EEG pattern characterized by "subclinical" spikes and waves occurring almost continuously during slow sleep and appearing every night for a variable length of time in children was reported under the title of "subclinical electrical status epilepticus induced by sleep in children". The disorder was later termed "electrical status epilepticus during sleep" [22]. The clinical manifestations of this syndrome include a status heterogeneous epileptic disorder, deterioration of neuropsychological functions associated with or independent from the epileptic disorder, and deterioration of motor functions. The typical EEG pattern of continuous spikes and waves during slow sleep (Figures 5 and 6) is also an essential and absolute feature for the recognition of the syndrome. The age at which the first seizure occurs ranges between 2 months and 12 years, with a peak around 4 and 5 years. This event can be preceded by either normal psychomotor development or abnormal signs indicating preexisting encephalopathy such as hemiparesis, hemiplegia, spastic quadriplegia, diffuse hypotonia, and ataxia. The seizure types occurring in the disorder can be both partial and generalized. They include unilateral or bilateral clonic seizures, generalized tonicclonic seizure, absences, partial motor seizures, complex partial seizures, or epileptic falls. They may occur during wakefulness or sleep. Tonic seizures, however, never occur. The first seizures are reported to be nocturnal and of unilateral type in almost one-half of the cases reported. At onset, the frequency of seizure attacks is low. At the time of discovery of the typical nocturnal EEG pattern, however, the epileptic seizures frequently change in severity and frequency. Absences and epileptic falls herald the appearance of continuous spikes and waves during slow sleep and seizure frequency increases, both during wakefulness and sleep. About $60 \%$ of patients also exhibit several types of seizures $[22,23]$. The characteristic feature of this disorder is the appearance of continuous spike-wave discharges on the EEG during slow sleep. Most researchers assert that more than $85 \%$ of NREM sleep is occupied by spike-wave discharges; however, quantitative studies of different sleep stages and of temporal evolution of this EEG disturbance have not been carried. The typical EEG changes appear 1 year to 2 years after the first seizure and are associated with behavioral deterioration. Focal and generalized interictal spikes occur before this time and persist during wakefulness and REM sleep after the appearance of continuous spike waves during slow-wave sleep [24]. The cause of electrical status epilepticus during slow sleep is unknown. Longlasting persistence of continuous spike waves during sleep is postulated to be responsible for the neuropsychiatric abnormalities in electrical status epilepticus during slow sleep; secondary bilateral synchrony is the mechanism underlying continuous spikes and waves during slow sleep. In this respect the apparent generalized seizures (absences, tonic-clonic attacks) occurring in this condition have in fact a focal onset as demonstrated by interhemispheric peak latencies of their EEG correlates, phase reversal of spikes on unilateral frontal regions, and studies of coherence and phase analyses. A localized metabolic abnormality has been also revealed by means of PET studies. Therefore, although electrical status epilepticus during slow sleep is currently classified among the epilepsies undetermined whether focal 
or generalized, consistent data support the view that this syndrome is to be included in the domain of localizationrelated epilepsies, of cryptogenic or symptomatic nature [23-25]. Electrical status epilepticus during slow sleep is a rare order. One study revealed an incidence of $0.5 \%$ among 12,854 children evaluated during a 10 -year period. There is no obvious gender preponderance [23]. The seizures in electrical status epilepticus during slow sleep are self-limited and disappear in the midteens. The good seizure outcome is independent of the etiology and is observed also in cases with cortical malformations such as multilobar polymicrogyria. The characteristic EEG patterns during slow-wave sleep also disappear at approximately the same time, but focal interictal spikes may persist. Improvement in language dysfunction, mental retardation, and psychiatric disturbances generally occurs but it is variable and individualized. The majority of affected children never return to normal levels, particularly in the verbal area and attention $[25,26]$. Epileptic seizures may or may not respond to a variety of drugs including benzodiazepines, valproate, ethosuximide, carbamazepine, and phenytoin. Despite of the fact that the seizures may be refractory to therapy for months to years, the long-term prognosis of epilepsy is favorable with the disappearance of seizures in all cases. Only benzodiazepines and adrenocorticotrophic hormone have been reported to suppress the electrical status and perhaps to improve language function. However, the positive effects are often transient [23]. In individual cases, chronic oral treatment with clobazam, lorazepam, and clonazepam associated with other antiepileptic drugs usually valproate seemed to have a long-lasting effect. Short cycles (3 to 4 weeks $)$ of relatively high doses of diazepam $(0.5 \mathrm{mg} / \mathrm{kg})$ following a rectal diazepam bolus of $1 \mathrm{mg} / \mathrm{kg}$ have also been reported to be effective. At the present time, the combined use of benzodiazepines and valproate is considered the treatment of choice in this condition. On the other hand, polytherapy should be avoided. A detailed evaluation of antiepileptic regimens in 88 patients demonstrated that the reduction in polytherapy coincided with an improvement of the syndrome. It was also suggested that the drug overload and some medications (such as carbamazepine) could play a role in the maintenance of continuous spikes and waves during slow sleep. In cases of electrical status epilepticus during sleep with severe language impairment, a progressive and long-lasting improvement of the language function has been obtained applying the surgical procedure of multiple subpial transections of the region of focal epileptic discharges [27].

3.3. Acquired Epileptic Aphasia Landau-Kleffner Syndrome (LKS). Acquired epileptic aphasia typically develops in healthy children who acutely or progressively lose receptive and expressive language ability coincident with the appearance of paroxysmal electroencephalographic (EEG) changes [28].

In most cases described in detail, a clearly normal period of motor and language development occurs before acquired epileptic aphasia symptoms appear. However, in the last 2-3 decades, several reported cases have been difficult to classify, because the patients' presenting symptoms appear to have

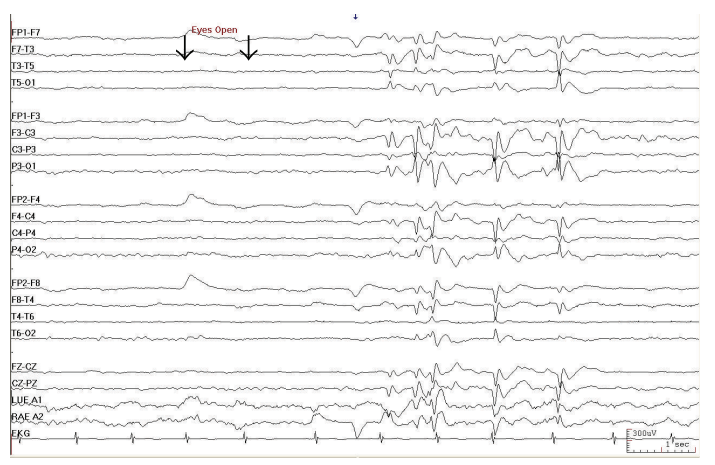

FIGURE 5: Electrical status epilepticus during slow sleep (ESES), disappearance of spike waves with eye opening (arrows).

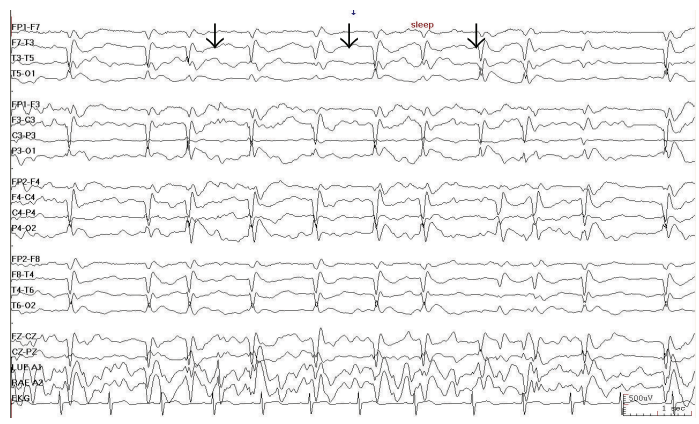

FIGURE 6: Electrical status epilepticus during slow sleep (ESES), continuous spike-wave discharges on the EEG during slow sleep (arrows).

variants of those originally described. In one case, expressive language deteriorated instead of receptive language, whereas in another case, a brief period of normal language development (single words) was followed by language regression with abnormal EEG findings. Acquired epileptic aphasia must be differentiated from autism with minimal language regression, especially when it is associated with isolated EEG abnormalities. Many current researchers classify acquired epileptic aphasia as part of the syndrome of electrical status epilepticus of sleep (ESES) [25]. Whether seizures and epileptiform discharges cause language dysfunction in acquired epileptic aphasia (AEA) is disputed. Aphasia and electroencephalographic (EEG) abnormalities might have a common cause (e.g., a left temporal brain astrocytoma or head injury). Some authors speculate that reinforcement of synaptogenesis mediates the neurologic deficits in acquired epileptic aphasia and that epileptiform discharges during a critical period of synaptic reinforcement or pruning in turn mediate the reinforcement of synaptogenesis [28]. In affected children, aphasia usually appears at age 4-7 years, and there is a slight male predominance (male-tofemale ratio, 1.7:1). However, symptom onset has been described in patients as young as 18 months and in those as old as 13 years. This discussion excludes the congenital cases with typical electroencephalographic (EEG) patterns and little or no language development; in such cases, 
the precise age of onset can never be determined. Longterm outcome studies of patients with acquired epileptic aphasia are limited by the lack of uniformity in diagnostic criteria. About half the patients have some fluctuation in aphasia, and the fluctuations usually occur over several months. On occasion, aphasia may worsen for as long as 7 years after the disease onset [29]. The treatment of acquired epileptic aphasia is far from standard, and many therapeutic modalities have been tried with variable success. Among these are anticonvulsant drugs, corticosteroids (e.g., adrenocorticotropin hormone (ACTH)), ketogenic diet, and surgical intervention with multiple subpial transections (MSTs). The calcium channel blocker nicardipine has been used in the treatment of acquired epileptic aphasia. In the initial report of 4 patients that suggested the use of nicardipine for acquired epileptic aphasia, nicardipine was given in association with anticonvulsant medications (carbamazepine, valproic acid) and corticosteroids (3 of 4 cases). However, cessation of nicardipine was associated with acute speech deterioration. The dose of nicardipine was $1 \mathrm{mg} / \mathrm{kg} / \mathrm{d}$ or $60 \mathrm{mg} / \mathrm{d}$ for large patients. A few case reports have demonstrated that intravenous gamma globulin may be useful in acquired epileptic aphasia, but repeated doses may be necessary [30]. Many commonly used anticonvulsant agents effective against partial or generalized seizures have been used in acquired epileptic aphasia with variable success. Phenobarbital, carbamazepine, and phenytoin are often ineffective in halting the electroencephalographic (EEG) discharges, and aphasia may worsen the electrographic activity. In a few cases, the drugs may actually worsen the picture, especially in patients with drop seizures and atypical absences. Valproic acid, ethosuximide, and benzodiazepines alone or in combination have been partially or transiently effective in some cases. Benzodiazepines, especially clobazam (in Europe) and midazolam, have been most effective when given intravenously (IV). Both the impracticality of this mode of administration and its short-lived effect have limited its use. Diazepam $0.5 \mathrm{mg} / \mathrm{kg}$ given rectally (PR) at bedtime is sometimes effective. This treatment is used in 4- to 6-week courses on and off to avoid tachyphylaxis. The Boston Children's Hospital Epilepsy Group has used continuous diazepam $0.5-0.3 \mathrm{mg} / \mathrm{kg}$ given orally (PO) in acquired epileptic aphasia for periods up to 1 year. Several studies have shown levetiracetam to be beneficial when used as monotherapy in the treatment of electrical status epilepticus of sleep (ESES), continuous spike wave in slowwave sleep (CSWS), and benign idiopathic focal epilepsies in childhood. In a recent study, Kramer et al. found clobazam and levetiracetam to be the most efficacious antiepileptic drugs in the treatment of ESES. In a case report, felbamate $45 \mathrm{mg} / \mathrm{kg} / \mathrm{d}$ was successful in treating seizures and aphasia. However, the high frequency of aplastic anemia and liver dysfunction with this drug limits its use [31,32].

\section{Conclusions}

Epileptic encephalopathies are severe brain disorders of early age that manifest with (1) electrographic EEG paroxysmal activity that is often aggressive, (2) seizures that are usually multiform and intractable, (3) cognitive, behavioral and neurological deficits that may be relentless, and (4) sometimes early death. The concept of "epileptic encephalopathies" is based on the assumption that aggressive ictal (seizure) and electrical (electrographic) epileptogenic activity during brain maturation is the main causative factor of progressive cognitive and neuropsychological deterioration or regression. Conversely, this deleterious epileptic activity is a specific age-related brain reaction of excessive neocortical excitability to different pathological conditions, which are focal or diffuse, of symptomatic or idiopathic cause. This age-related epileptogenic reaction is peculiar to the immature brain and varies significantly in accordance with the stage of brain maturity at the time that this occurs. Thus, EEG demonstrates primarily burst-suppression patterns in the neonatal period, hypsarrhythmia in infancy, and slow generalized spike-wave discharges (GSWDs) in early childhood. With advancing age, the seizure and electrographic epileptogenic features may evolve from one to another age-related stage that is from burst suppression to hypsarrhythmia and then to slow GSWD. All epileptic encephalopathies have a tendency to abate, discontinue, or even stop in adolescence but often with serious neurocognitive residuals.

\section{References}

[1] J. Engel Jr., "A proposed diagnostic scheme for people with epileptic seizures and with epilepsy: report of the ILAE task force on classification and terminology," Epilepsia, vol. 42, no. 6, pp. 796-803, 2001.

[2] Y. Yamatogi and S. Ohtahara, "Multiple independent spike foci and epilepsy, with special reference to a new epileptic syndrome of 'severe epilepsy with multiple independent spike foci," Epilepsy research., vol. 70, pp. S96-104, 2006.

[3] J. F. Donat, "The age-dependent epileptic encephalopathies," Journal of Child Neurology, vol. 7, no. 1, pp. 7-21, 1992.

[4] J. Aicardi and S. Ohtahara, "Severe neonatal epilepsies with suppression-burst pattern," in Epileptic Syndromes in Infancy, Childhood and Adolescence, J. Roger, M. Bureau, C. H. Dravet, P. Genton, C. A. Tassinari, and P. Wolf, Eds., pp. 33-44, John Libbey, London, UK, 3rd edition, 2002.

[5] L. Fusco, C. Pachatz, M. Di Capua, and F. Vigevano, "Video/EEG aspects of early-infantile epileptic encephalopathy with suppression-bursts (Ohtahara syndrome)," Brain and Development, vol. 23, no. 7, pp. 708-714, 2001.

[6] N. Murakami, Y. Ohtsuka, and S. Ohtahara, "Early infantile epileptic syndromes with suppression-bursts: early myoclonic encephalopathy vs. Ohtahara syndrome," Japanese Journal of Psychiatry and Neurology, vol. 47, no. 2, pp. 197-200, 1993.

[7] H. Komaki, K. Sugai, M. Sasaki et al., "Surgical treatment of a case of early infantile epileptic encephalopathy with suppression-bursts associated with focal cortical dysplasia," Epilepsia, vol. 40, no. 3, pp. 365-369, 1999.

[8] J. Aicardi and S. Ohtahara, "Severe neonatal epilepsies with suppression-burst pattern," in Epileptic Syndromes in Infancy, Childhood and Adolescence, M. Bureau, C. H. Dravet, P. Genton, C. A. Tassinari, and P. Wolf, Eds., pp. 33-44, John Libbey, London, UK, 3rd edition, 2002. 
[9] B. D. Bernardina, O. Dulac, and N. Fejerman, "Early myoclonic epileptic encephalopathy (E.M.E.E.)," European Journal of Pediatrics, vol. 140, no. 3, pp. 248-252, 1983.

[10] S. Ohtahara and Y. Yamatogi, "Epileptic encephalopathies in early infancy with suppression-burst," Journal of Clinical Neurophysiology, vol. 20, no. 6, pp. 398-407, 2003.

[11] M. T. Mackay, S. K. Weiss, T. Adams-Webber et al., "Practice parameter: medical treatment of infantile spasms: report of the American Academy of Neurology and the Child Neurology Society," Neurology, vol. 62, no. 10, pp. 1668-1681, 2004.

[12] R. Caraballo, M. Vacarezza, R. Cersósimo et al., "Longterm follow-up of the ketogenic diet for refractory epilepsy: multicenter Argentinean experience in 216 pediatric patients," Seizure, 2011.

[13] F. Vigevano, L. Fusco, R. Cusmai et al., "The idiopathic form of West syndrome," Epilepsia, vol. 34, no. 4, pp. 743-746, 1993.

[14] G. Coppola, "Malignant migrating partial seizures in infancy: an epilepsy syndrome of unknown etiology," Epilepsia, vol. 50, supplement 5, pp. 49-51, 2009.

[15] B. D. Bernardina, E. Fontana, and F. Darra, "Myoclonic status in non progressive encephalopathies," in Advances in Neurology, A. Delgado-Escueta, R. Guerrini, M. T. Medina, P. Genton, M. Bureau, and C. Dravet, Eds., vol. 95 of Myoclonic Epilepsies, pp. 59-70, 2005.

[16] R. Guerrini, L. Parmeggiani, A. Kaminska, and O. Dulac, "Myoclonic astatic epilepsy," in Epilepsy Syndromes in Infancy, Childhood and Adolescence, J. Roger, M. Bureau, C. H. Dravet, P. Genton, C. A. Tassinari, and P. Wolf, Eds., pp. 106-112, John Libbey, London, UK, 3rd edition, 2002.

[17] R. Guerrini, C. Dravet, P. Genton, A. Belmonte, A. Kaminska, and O. Dulac, "Lamotrigine and seizure aggravation in severe myoclonic epilepsy," Epilepsia, vol. 39, no. 5, pp. 508-512, 1998.

[18] A. Arzimanoglou, J. French, W. T. Blume et al., "LennoxGastaut syndrome: a consensus approach on diagnosis, assessment, management, and trial methodology," The Lancet Neurology, vol. 8, no. 1, pp. 82-93, 2009.

[19] H. Heiskala, "Community-based study of Lennox-Gastaut syndrome," Epilepsia, vol. 38, no. 5, pp. 526-531, 1997.

[20] K. van Rijckevorsel, "Treatment of Lennox-Gastaut syndrome: overview and recent findings," Neuropsychiatric Disease and Treatment, vol. 4, no. 6, pp. 1001-1019, 2008.

[21] G. Kluger, G. Kurlemann, E. Haberlandt et al., "Effectiveness and tolerability of rufinamide in children and adults with refractory epilepsy: first European experience," Epilepsy and Behavior, vol. 14, no. 3, pp. 491-495, 2009.

[22] C. A. Tassinari, "The problems of 'continuous spikes and waves during slow sleep' or 'electrical status epilepsticus during slow sleep' today," in Continuous Spikes and Waves during Slow Sleep, A. Beaumanoir, M. Bereau, T. Deonna, L. Mira, and C. A. Tssinari, Eds., pp. 251-255, John Libbey, London, UK, 1995.

[23] M. Bureau, "Continuous spikes and waves during slow sleep (CSWS): definition of the syndrome," in Continuous Spikes and Waves during Slow Sleep or ESES, A. Beaumanoir, M. Bureau, T. Deonna, L. Mira, and C. A. Tassinari, Eds., pp. 1726, John Libbey, London, UK, 1995.

[24] K. Kobayashi, N. Nishibayoshi, Y. Ohtsuka, E. Oka, and S. Ohtahara, "Epilepsy with electrical status epilepticus during slow sleep and secondary bilateral synchrony.," Epilepsia, vol. 35, pp. 1097-1103, 1994.
[25] F. Morrell, "Electrophysiology of CSWS in Landau-Kleffner syndrome," in Continuous Spikes and Waves during Slow Sleep, A. Beaumanoir, M. Bureau, T. Deonna, L. Mira, and C. A. Tassinari, Eds., pp. 77-90, John Libbey, London, UK, 1995.

[26] T. Morikawa, M. Seino, Y. Watanabe, M. Watanabe, and K. Yagi, "Clinical relevance of continuous spike-waves during slow-wave sleep," in Proceedings of the 17th Epilepsy International Symposium on Advances in Epileptology, J. Manelis, E. Bental, J. N. Loeber, and F. E. Dreifuss, Eds., pp. 359-363, Raven Press, New York, NY, USA.

[27] M. De Negri, "Electrical status epilepticus during sleep (ESES). Different clinical syndromes: towards a unifying view?" Brain and Development, vol. 19, no. 7, pp. 447-451, 1997.

[28] C. J. Feekery, B. Parry-Fielder, and I. J. Hopkins, "LandauKleffner syndrome: six patients including discordant monozygotic twins," Pediatric Neurology, vol. 9, no. 1, pp. 49-53, 1993.

[29] M. H. C. Duran, C. A. Guimarães, L. L. Medeiros, and M. M. Guerreiro, "Landau-Kleffner syndrome: long-term follow-up," Brain and Development, vol. 31, no. 1, pp. 58-63, 2009.

[30] W. F. M. Arts, F. K. Aarsen, M. Scheltens-De Boer, and C. E. Catsman-Berrevoets, "Landau-Kleffner syndrome and CSWS syndrome: treatment with intravenous immunoglobulins," Epilepsia, vol. 50, supplement 7, pp. 55-58, 2009.

[31] J. J. Riviello, “The Boston Children's Hospital experience with ESES and LKS course," in Annual Meeting of the American Epilepsy Society, San Diego, Calif, December 2006.

[32] C. von Stülpnagel, G. Kluger, S. Leiz, and H. Holthausen, "Levetiracetam as add-on therapy in different subgroups of 'benign' idiopathic focal epilepsies in childhood," Epilepsy and Behavior, vol. 17, no. 2, pp. 193-198, 2010. 


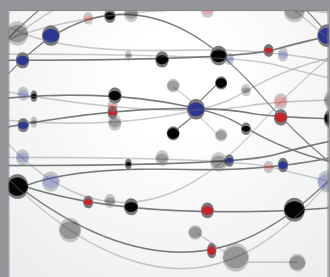

The Scientific World Journal
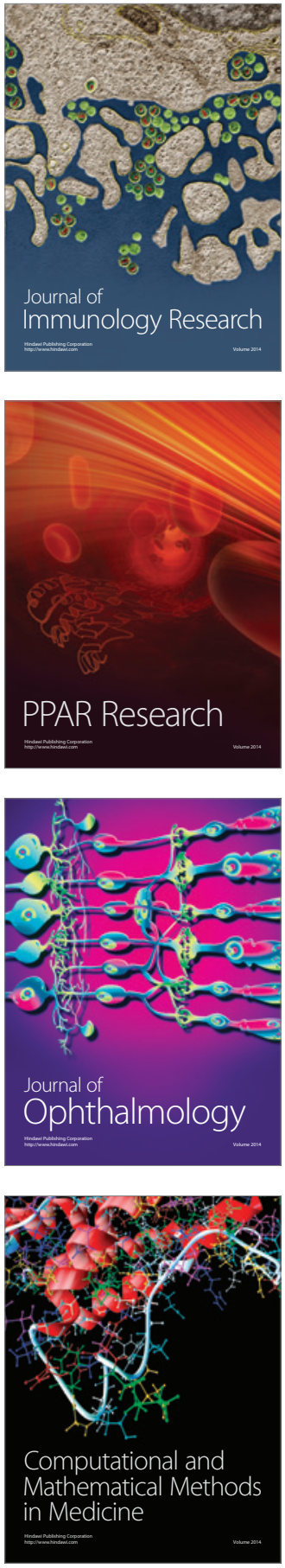

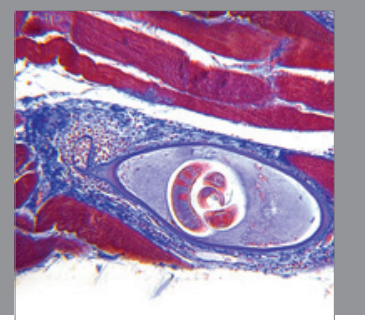

Gastroenterology

Research and Practice
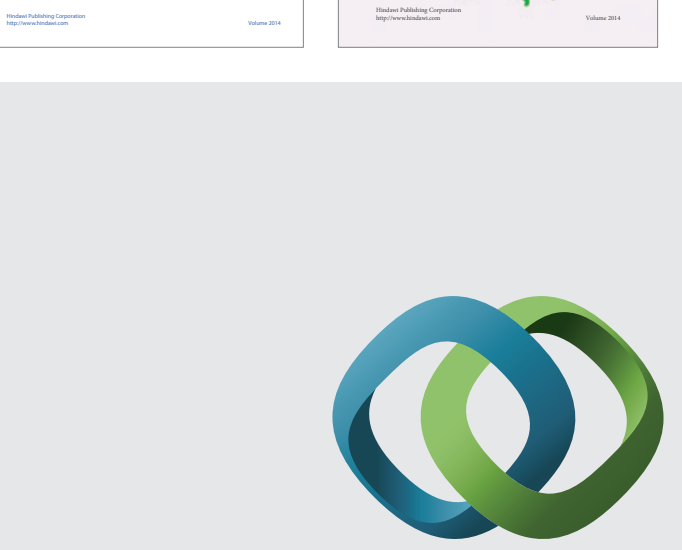

\section{Hindawi}

Submit your manuscripts at

http://www.hindawi.com
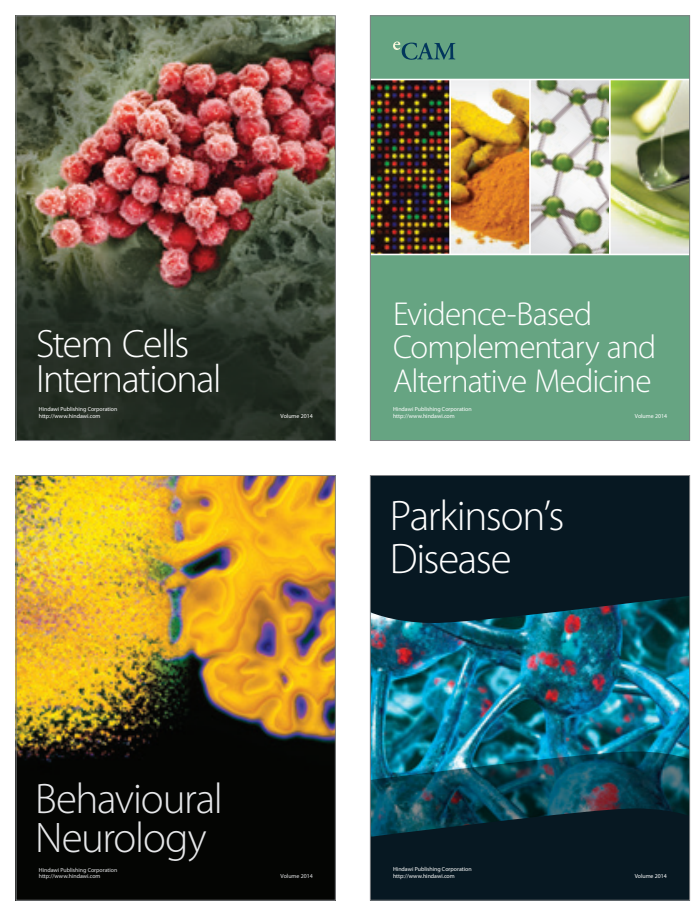

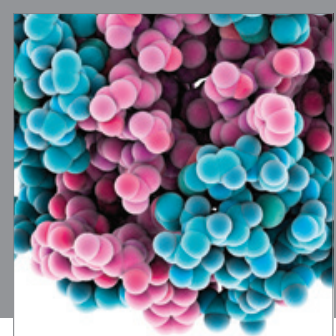

Journal of
Diabetes Research

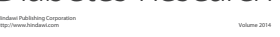

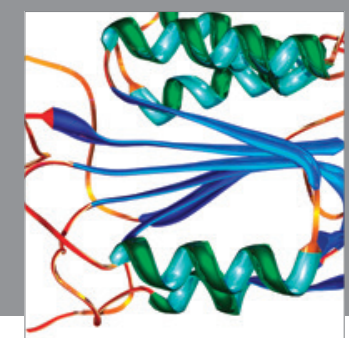

Disease Markers
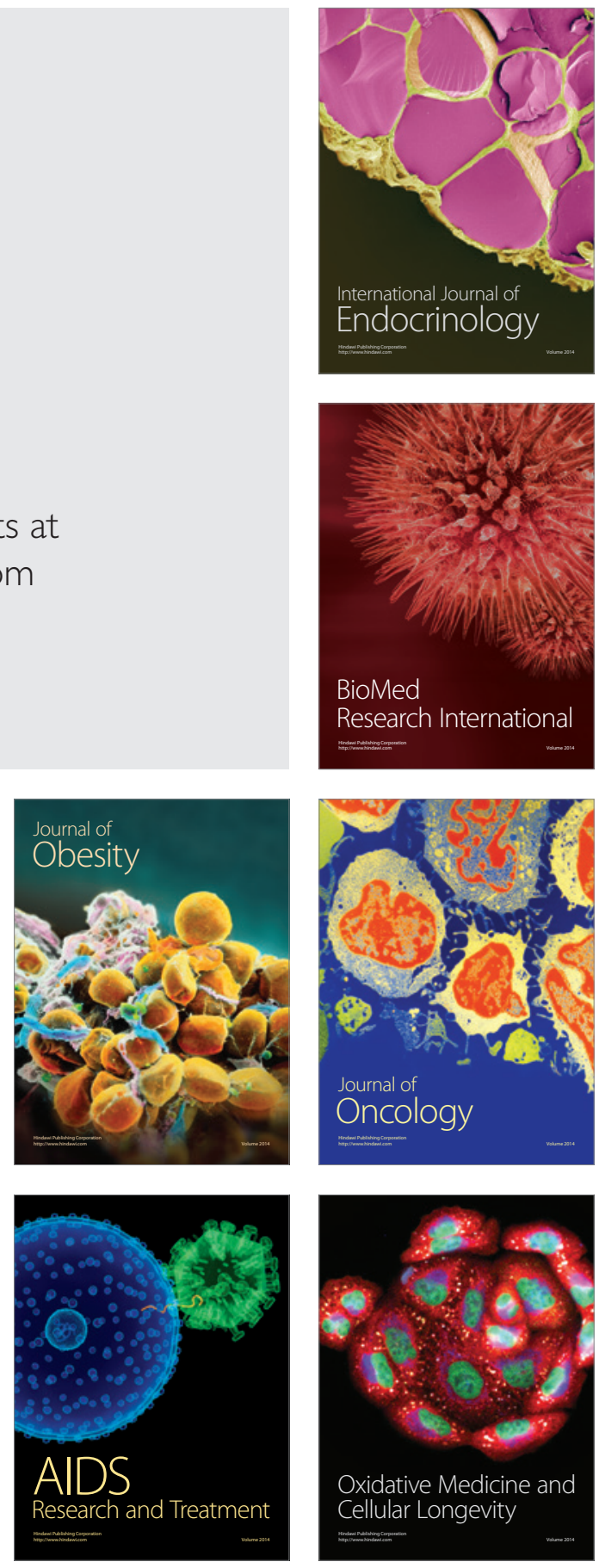